\title{
A Comparative Study on Modeling Methods for Switched Reluctance Machines
}

\author{
Shoujun Song \& Weiguo Liu \\ School of Automation, Northwestern Polytechnical University \\ PO box 352, 127 Youyi Xilu, Xi'an, 710072, Shaanxi, China \\ Tel: 86-29-8843-1309 E-mail: songshoujun@hotmail.com
}

\begin{abstract}
Switched reluctance motor (SRM) has many specific advantages and has being gradually used in all kinds of applications. However, because it's inherently multivariable, strong-coupling and highly nonlinear, there are lots of difficulties to build its model. In this paper, many modeling methods include numerical, analytical and intelligent approaches are studied and compared in terms of complexity and accuracy. The static flux linkage and torque characteristics obtained by each method are given. The advantages and disadvantages of each method are summarized as well.
\end{abstract}

Keywords: Switched reluctance motor (SRM), Modeling methods, Finite element analysis (FEA), Fourier decomposition (FD), Back propagation neural network (BPNN)

\section{Introduction}

The SRM has the definite advantages of simple magnetless and rugged construction, simple control, ability of extremely high speed operation, relatively wide constant power capability, minimal effects of temperature variations offset, low manufacturing cost and ability of hazard-free operation (W. Xu et al., 2009). These distinct advantages make the SRM well suited to kinds of applications, such as more/all electric aircrafts (S. Song, 2009) and electric vehicles (X. D. Xue et al., 2008).

In contrast to traditional motors (such as induction motors), the SRM is intended to operate in deep magnetic saturation to increase the output power density and the energy conversion factor. Therefore, because of saturation effects and the variation of magnetic reluctance, all the relevant characteristics (i.e., flux linkage, torque) of the machines are highly nonlinear functions of both rotor position and phase current (R. T. Naayagi et al., 2005). These nonlinearities make the modeling process of the SRM relatively difficult.

Due to saturation effects in the SRM, establishing the accurate nonlinear mapping relationship of the flux linkage with respect to phase current and rotor position is the basis of performance calculation (J. Xiu et al., 2007). For years, many modeling methods have been proposed by worldwide researchers (O. Ichinokura et al., 2003 ; S. Song et al., 2007; Z. Lin et al., 2007). Generally speaking, these methods can be divided into three categories: numerical, analytical and intelligent approaches. They have advantages and disadvantages of their own.

In this paper, three typical methods belong to different categories are studied respectively and their performances are compared in terms of complexity and accuracy. These three methods are based on finite element analysis (FEA), Fourier decomposition (FD) and back propagation neural network (BPNN).

\section{Numerical methods}

Among numerous numerical methods, FEA is the most frequently used approach (K. N. Srinivas et al., 2005). There are many softwares that can be used to analyze and calculate the electromagnetic field of the motor accurately in two-dimension (2-D) and three-dimension (3-D) ways. Before getting the static characteristics and dynamic performances of the motor, following steps need to be completed firstly.

(1) Build the model of the motor according to its physical dimension and material.

(2) Build the model of the power converter according to its topology.

(3) Set the terminal and boundary conditions.

(4) Add driving sources.

(5) Generate the finite element mesh.

Fig. 1 shows the 2-D FEA model of a four-phase 8/6-pole SRM. Fig. 2 shows the model of an asymmetric half bridge power converter, where S1 S8 are voltage-controlled switches, they represent the power switches in the converter. W1 W8 are current-controlled switches, they are used to implement current chopping control. Fig. 3 
shows the finite element mesh of the SRM.

After the completion of above steps, the electromagnetic characteristics of the motor can be obtained. Fig. 4 shows the magnetic field distribution inside the motor at one position. Fig. 5 shows the static flux linkage and torque characteristics of the SRM obtained by FEA. It should be noted that the FEA only can get some discrete points (represented by circles in the figure) and the curves are obtained by the "plot" function of the MATLAB to indicate the variation trend of the data.

\section{Analytical methods}

In analytical methods, the nonlinear relationships of the phase inductance and torque to phase current and rotor position are represented by several analytical expressions. Among numerous analytical methods, the linear and quasi-linear models are well-known. In this paper, a method called FDM is studied. It's based on Fourier decomposition (FD) (J. Hur et al., 2003).

It is well known that the phase inductance curve of the SRM is a periodic function, so it can be expanded in Fourier series as shown in Eq. (1).

$$
L(\theta, i)=L_{0}(i)+\sum_{m=1}^{n}\left[L_{m}(i) \cos \left(m N_{r} \theta\right)\right]
$$

where $L(\theta, i)$ is the phase inductance, $L_{0}(i)$ and $L_{m}(i)$ are coefficients, $N_{r}$ is the number of rotor poles, $\theta$ is the angular position of the rotor, $m=[1, n]$ is the harmonic coefficient, $n$ is the maximum harmonic order.

The modeling accuracy is strongly affected by $n$. In this paper, $n$ is equal to 1 . In this case Eq. (1) can be rewritten as

$$
L(\theta, i)=L_{0}(i)+L_{1}(i) \cos \left(N_{r} \theta\right)
$$

To calculate the two undetermined coefficients in Eq. (2), the inductance values at two different rotor positions are needed. In this paper, $0^{\circ}$ and $30^{\circ}$ (see Fig. 5(a)), namely unaligned and aligned position, are selected. Substituting the inductance values at these two positions into Eq. (2), following equations can be obtained.

$$
\left\{\begin{array}{l}
L\left(0^{\circ}, i\right)=L_{\min }=L_{0}(i)+L_{1}(i) \\
L\left(30^{\circ}, i\right)=L_{\max }=L_{0}(i)-L_{1}(i)
\end{array}\right.
$$

where $L_{\min }$ and $L_{\max }$ are inductance values at unaligned and aligned positions, respectively.

Those two undetermined coefficients in Eq. (2) can be easily obtained from Eq. (3), as

$$
\left\{\begin{array}{l}
L_{0}(i)=\left(L_{\min }+L_{\max }\right) / 2 \\
L_{1}(i)=\left(L_{\min }-L_{\max }\right) / 2
\end{array}\right.
$$

It is well known that $L_{\min }$ can be seen as a constant, while $L_{\max }$ changes along with the phase current nonlinearly. The analytical expression between $L_{\max }$ and phase current $i$ can be obtained by polynomial fitting, as

$$
L_{\max }(i)=\sum_{m=0}^{n} a_{m} i^{m}
$$

where $a_{m}$ are polynomial coefficients that can be obtained by fitting.

The data of phase inductance at aligned position with different phase currents needed for polynomial fitting can be easily obtained from FEA results (see Fig. 5(a)) by following equation.

$$
L\left(i_{0}, \theta_{0}\right)=\frac{\psi\left(i_{0}, \theta_{0}\right)}{i_{0}}
$$

where $L\left(i_{0}, \theta_{0}\right)$ and $\psi\left(i_{0}, \theta_{0}\right)$ are phase inductance and flux linkage at $\theta_{0}$ with phase current equal to $i_{0}$, respectively.

The analytical expressions of those two undetermined coefficients in Eq. (2) can be obtained by substituting Eq. (5) and the value of $L_{\min }$ into Eq. (4). Finally, the phase inductance can be expressed as

$$
L(\theta, i)=\left(L_{\min }+\sum_{m=0}^{n} a_{m} i^{m}\right) / 2+\left(L_{\min }-\sum_{m=0}^{n} a_{m} i^{m}\right) \cos \left(N_{r} \theta\right) / 2
$$

Based on Eq. (7), the torque can be expressed as 


$$
T=\partial W_{\text {mag }}^{\prime} /\left.\partial \theta\right|_{i=c}=\partial \int_{0}^{i} L(\theta, i) i d i / \partial \theta=-\frac{N_{r} i^{2}}{4}\left(L_{\min }-\sum_{m=0}^{n} \frac{2 a_{m}}{m+2} i^{m}\right) \sin \left(N_{r} \theta\right)
$$

where $T$ is the torque, $W_{m a g}^{\prime}$ is the magnetic co-energy.

On the basis of Eq. (6) (8), the static flux linkage and torque characteristics of the SRM can be obtained as shown in Fig. 6.

\section{Intelligent methods}

There are many intelligent methods that can be used to build the model of SRM, such as artificial neural network (ANN) and fuzzy inference system (FIS). ANN has excellent capacity of nonlinear mapping, adaptive learning and parallel processing. In this paper, the back propagation neural network (BPNN) based on Levenberg-Marquardt (LM) optimization algorithm (Y. Cai, 2006; J. Sun et al., 2005) are used to implement the nonlinear mapping relationship of the flux linkage and torque to phase current and rotor position, namely $i(\psi, \theta)$ and $T(i, \theta)$. This method has strong robustness and adaptive ability and the user does not need have any professional knowledge about the SRM.

The data needed to train the BPNN can be obtained by FEA (see Fig. 5). To verify the approximation and generalization ability of the trained BPNN, the data in Fig. 5 are divided into two parts. In Fig. 5(a), the data with even rotor position values are used to train $i(\psi, \theta)$. In Fig. 5(b), the data with even phase current values are used to train $T(i, \theta)$.

In this paper, the BPNN used to train the $i(\psi, \theta)$ has two inputs ( $\psi$ and $\theta)$, one output $(i)$ and two hidden layers, both hidden layers have ten neurons. The BPNN used to train the $T(i, \theta)$ also has two inputs $(i$ and $\theta)$, one output $(T)$ and two hidden layers, both hidden layers have eight neurons.

Fig. 7 shows the variation of the error along with the epochs during training. It can be seen that the convergence speeds of both BPNN are fast.

Fig. 8 shows the comparison between the simulation results of BPNN and the data from FEA. All the FEA data in this figure are used to train the BPNN. It can be seen that both trained BPNN have strong approximation ability.

Fig. 9 shows the comparison between the simulation results of BPNN and the data from FEA. All the FEA data in this figure are NOT used in the training of the BPNN. It can be seen that both trained BPNN have strong generalization ability.

\section{Comparison of the modeling methods}

The numerical methods can get the static characteristics and dynamic performances of the SRM accurately, but quite complex and time-consuming. Furthermore, it's relatively difficult to implement the control algorithms by FEA softwares. The modeling and computational time of analytical methods is much shorter than that required by numerical methods. However, because of the introduction of many hypotheses and simplifications, the modeling accuracy of analytical methods is worse than that of numerical methods (see Fig. 6). The intelligent methods can find the reasonable balance between modeling time and accuracy.

According to the above-mentioned advantages and disadvantages of each modeling method, the numerical methods are commonly used to verify the performances of the designed motors in detail. The analytical methods are commonly used to briefly study the operating characteristics of the motor and quickly evaluate the performances of different control algorithms. The intelligent methods can be used to some applications in which repetitive computations are required, such as design optimization and dynamic performance analysis.

\section{Conclusion}

In this paper, three categories of modeling methods, namely numerical, analytical and intelligent approaches, are studied and compared in terms of complexity and accuracy. Three typical methods respectively based on FEA, FD and BPNN are given as examples. The simulation results show that they have advantages and disadvantages of their own. The numerical methods can get the static characteristics and dynamic performances of the SRM accurately, but quite complex and time-consuming. The modeling and computational time of analytical methods is much shorter than that required by numerical methods. However, its modeling accuracy is worse. The intelligent methods can find the reasonable balance between modeling time and accuracy. For different applications, the modeling methods should be selected carefully according to the features of the application and the advantages and disadvantages of the modeling methods. 


\section{References}

J. Hur, C. Kim and D. Hyun. (2003). Modeling of switched reluctance motor using Fourier series for performance analysis. Journal of Applied Physics, vol. 93, no. 10, May, pp. 8781-8783.

J. Sun, Q. Zhan and L. Liu. (2005). Modelling and control of bearingless switched reluctance motor based on artificial neural network. Proceedings of 31st Annual Conference of IEEE Industrial Electronics Society, Raleigh, NC, United States, Nov., pp. 1638-1643.

J. Xiu and C. Xia. (2007). Modeling of Switched Reluctance Motor Based on GA Optimized T-S Type Fuzzy Logic. Proceedings of Fourth International Conference on Fuzzy Systems and Knowledge Discovery, Haikou, China, pp. 186-191.

K. N. Srinivas and R. Arumugam. (2005). Analysis and Characterization of Switched Reluctance Motors: Part II - Flow, Thermal, and Vibration Analyses. IEEE Transactions on Magnetics, vol. 41, no. 4, Apr., pp. 1321-1332.

O. Ichinokura, T. Kikuchi, K. Nakamura, T. Watanabe and H. Guo. (2003). Dynamic Simulation Model of Switched Reluctance Generator. IEEE Transactions on Magnetics, vol. 39, no. 5, Sept., pp. 3253-3255.

R. T. Naayagi and V. Kamaraj. (2005). A Comparative Study of Shape Optimization of SRM using Genetic Algorithm and Simulated. Proceedings of IEEE Indicon 2005 Conference, Chennai, India, Dec., pp. 596-599.

S. Song, W. Liu and Y. Wang. (2007). Modeling, dynamic simulation and control of a four-phase switched reluctance motor. Proceedings of IEEE International Conference on Control and Automation, Guangzhou, China, May, pp. 1290-1295.

S. Song. (2009). Detailed design of a $30 \mathrm{~kW}$ switched reluctance starter/generator system used in more/all electric aircraft. Aachen, Germany: Shaker Verlag.

W. Xu, J. Zhu, Y. Guo, S. Wang, Y. Wang and Z. Shi. (2009). Survey on electrical machines in electrical vehicles. Proceedings of International Conference on Applied Superconductivity and Electromagnetic Devices, Chengdu, China, Sept., pp. 167-170.

X. D. Xue, K. W. E. Cheng and N. C. Cheung. (2008). Selection of Electric Motor Drives for Electric Vehicles. Proceedings of 2008 Australasian Universities Power Engineering Conference, Sydney, NSW, Dec., pp. 1-6.

Y. Cai. (2006). Research on Switched Reluctance Motor Nonlinear Modeling and High-Performance System. Ph.D. thesis, Tianjin University, Tianjin, China.

Z. Lin, D. S. Reay, B. W. Williams and X. He. (2007). Online modeling for switched reluctance motors using b-spline neural networks. IEEE Trans on Industrial Electronics, vol. 54, no. 6, Dec., pp. 3317-3322.

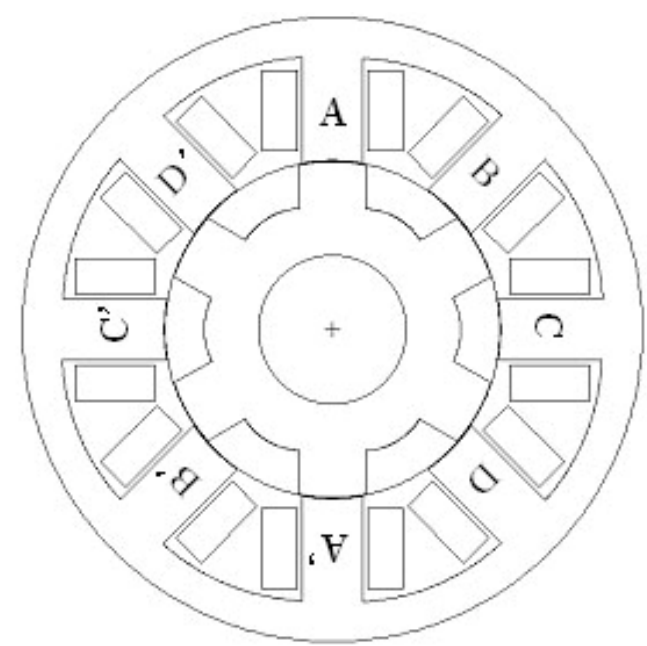

Figure 1. The 2-D FEA model of a four-phase 8/6-pole SRM 


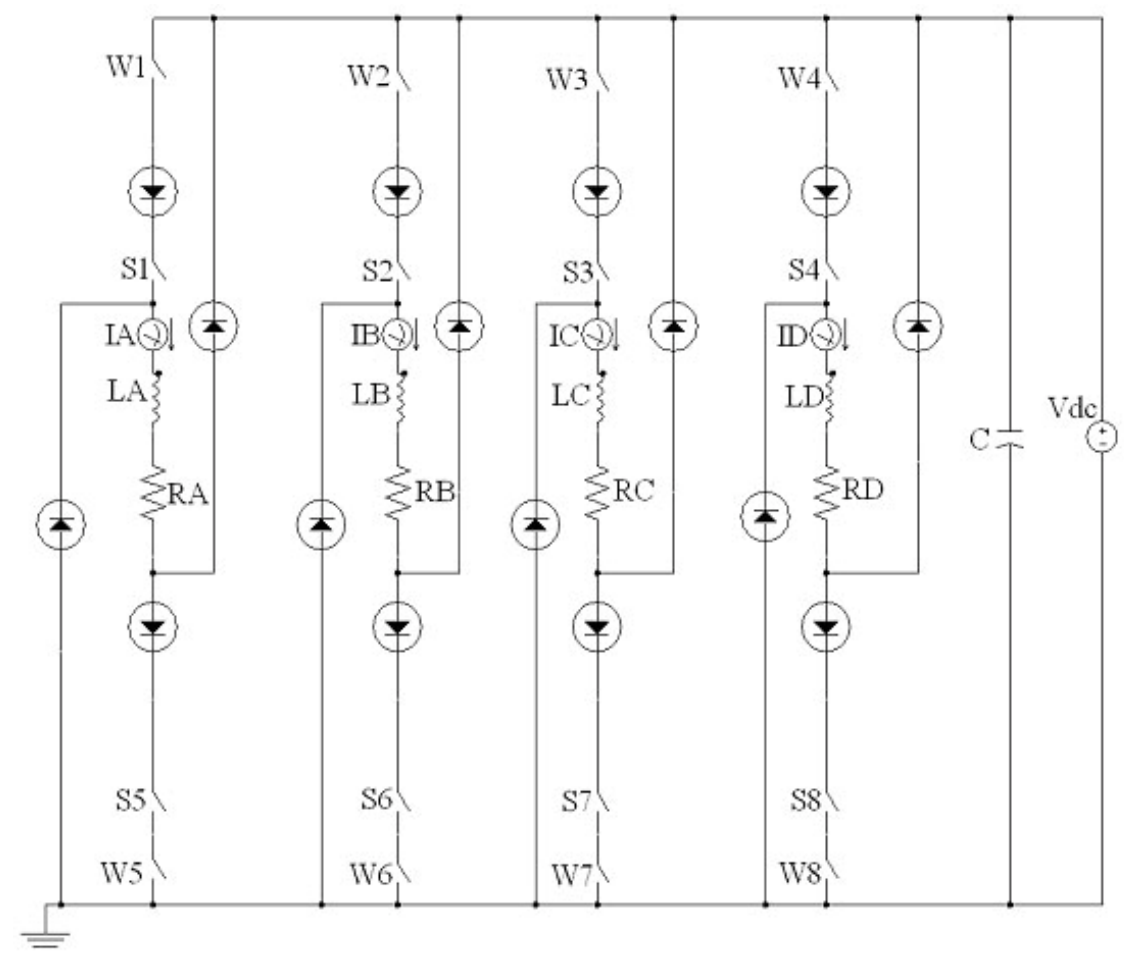

Figure 2. Model of an asymmetric half bridge power converter

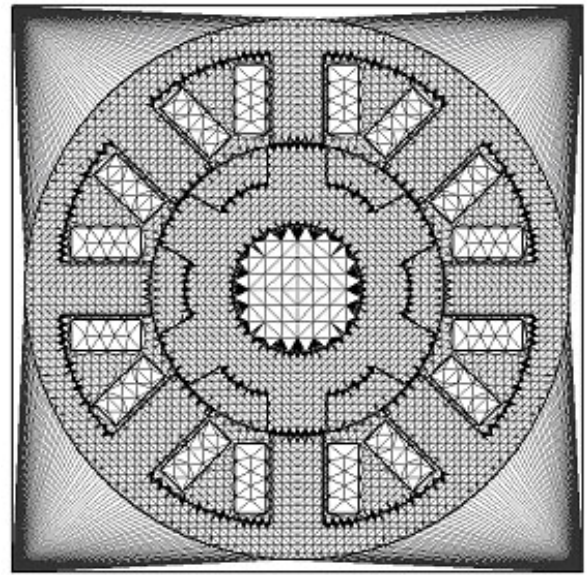

Figure 3. Finite element mesh of the SRM

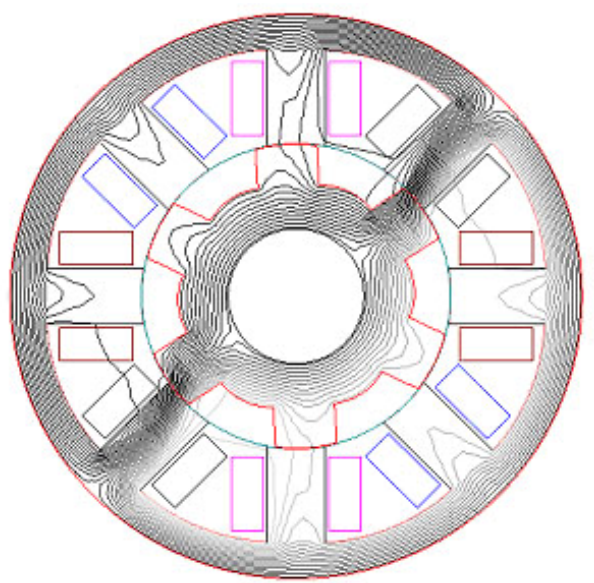

Figure 4. The magnetic field distribution inside the motor

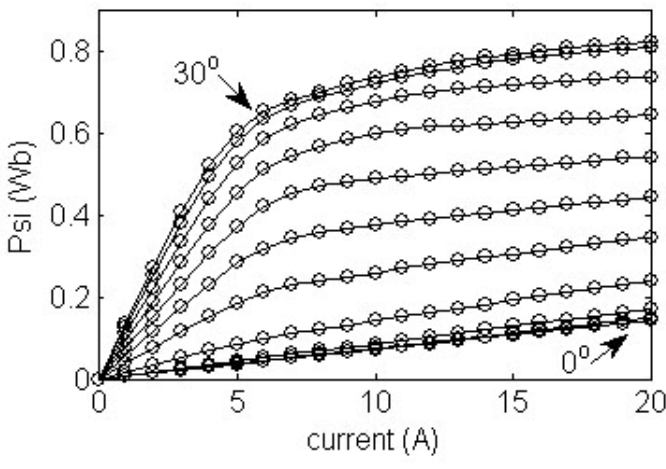

(a) Flux linkage characteristics

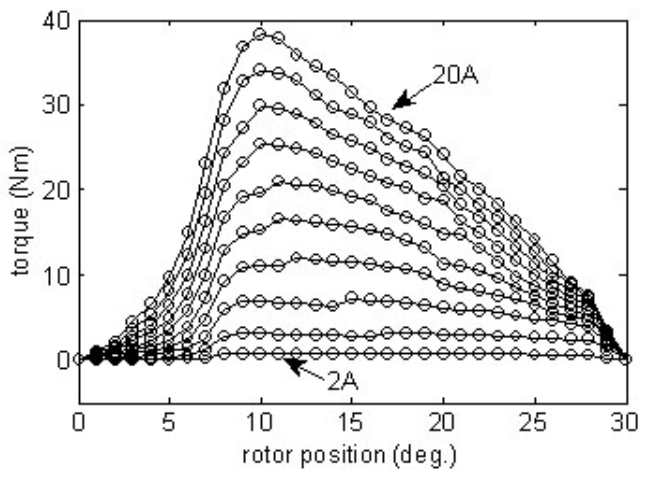

(b) Torque characteristics

Figure 5. Flux linkage and torque characteristics obtained by FEA 


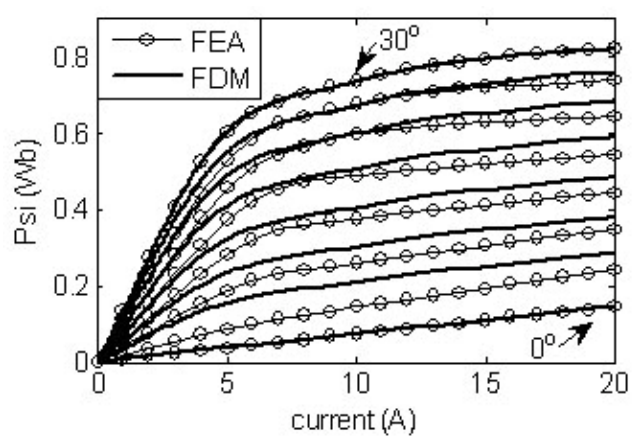

(a) Flux linkage characteristics

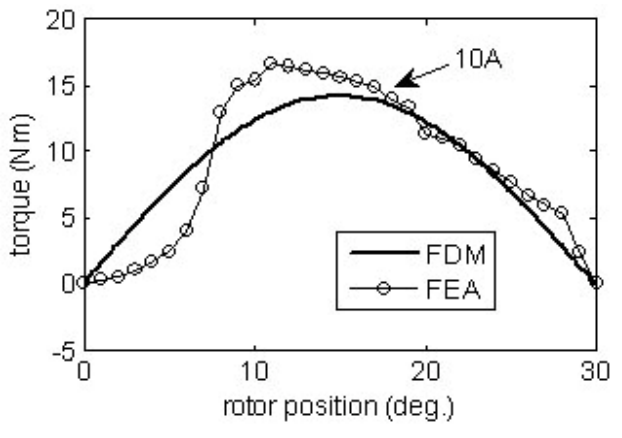

(b) Torque characteristics

Figure 6. Comparison between FEA and FDM

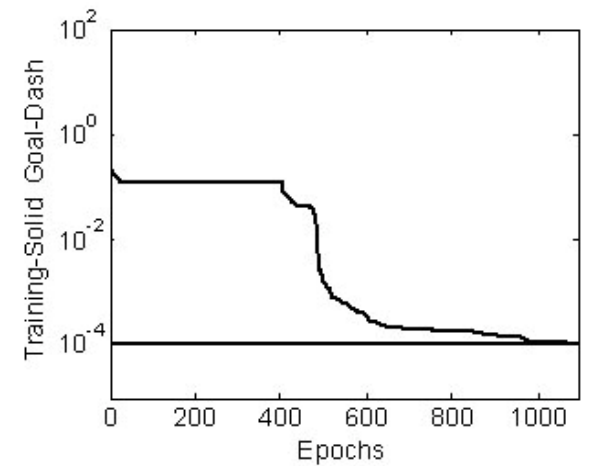

(a) $i(\psi, \theta)$

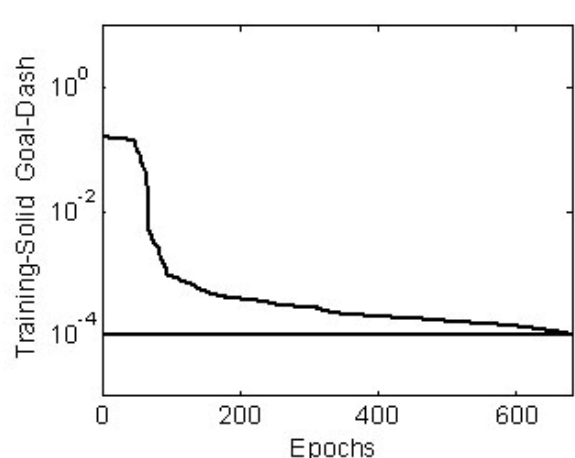

(b) $T(i, \theta)$

Figure 7. Variation of the error along with the epochs

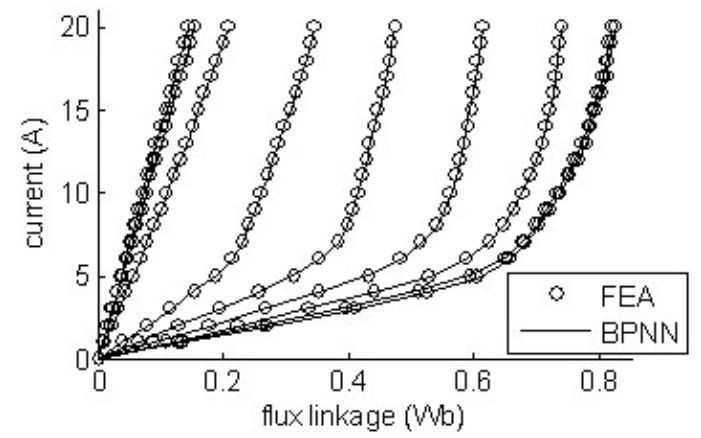

(a) $i(\psi, \theta)$

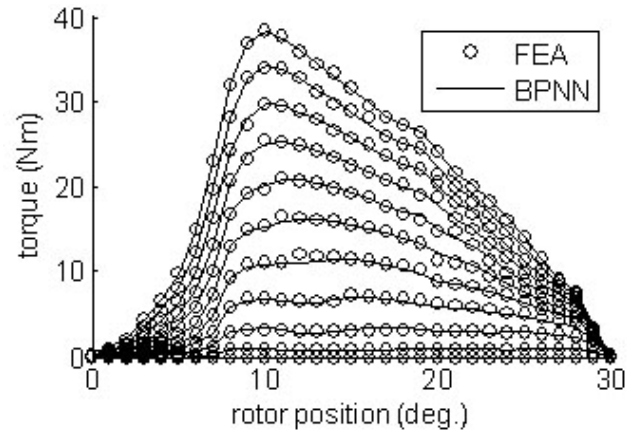

(b) $T(i, \theta)$

Figure 8 . Verification of the approximation ability

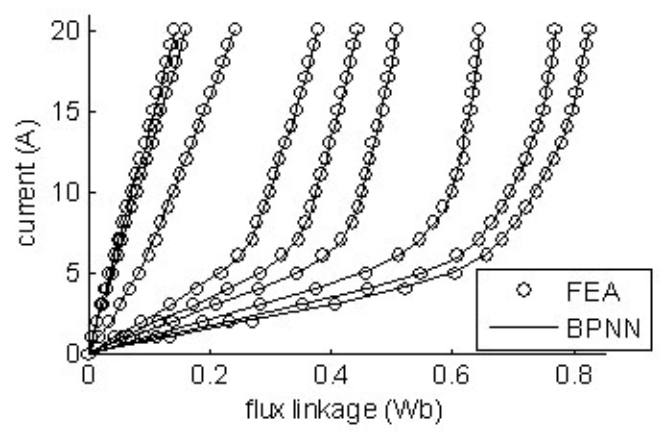

(a) $i(\psi, \theta)$

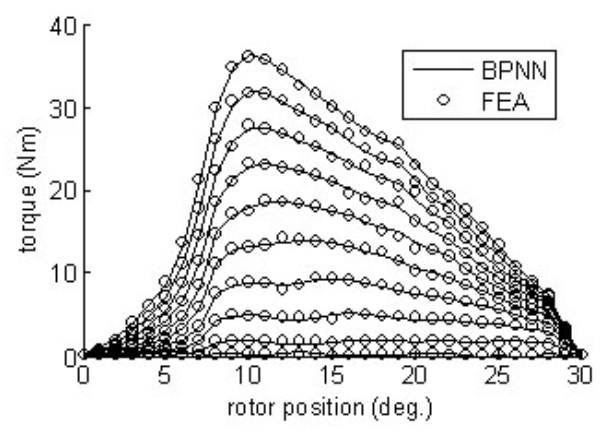

(b) $T(i, \theta)$

Figure 9 . Verification of the generalization ability 